\title{
ESTUDO DAS PROPORÇÕES MOLARES DE ETANOL NAS PROPRIEDADES DA PENEIRA MOLECULAR SBA-
} 15

\author{
F. O. $\operatorname{COSTA}^{1}$, C. G. A. MISAEL ${ }^{2}$, A. M. da $\operatorname{SILVA}^{3}$ e B. V. de SOUSA ${ }^{4}$ \\ ${ }^{1}$ Universidade Federal de Campina Grande, Departamento de Engenharia Química. \\ francielecostapb@gmail.com
}

\begin{abstract}
RESUMO - A sílica SBA-15 possui poros altamente ordenados em mesocanais dispostos na forma hexagonal e pode ser sintetizada numa larga faixa de composições. O etanol é um solvente polar prótico totalmente miscível em água. No processo de cristalização, o álcool acelera o desprendimento dos núcleos impedindo a formação do precipitado, agindo na superfície e reduzindo o tempo de síntese. O presente trabalho tem como objetivo sintetizar essa peneira molecular em diferentes porporções de cosolvente, etanol. A SBA-15 foi sintetizada utilizando como direcionador estrutural o copolímero tribloco não-iônico Pluronic P123 $\left(\mathrm{EO}_{20} \mathrm{PO}_{70} \mathrm{EO}_{20}\right)$ e como fonte de silício o tetraetilortosilicato (TEOS), em meio fortemente ácido $(\mathrm{pH}<2)$. Diante dos difratogramas e análise textural, verificou-se que o etanol melhorou a organização da estrutura da peneira molecular SBA-15. Assim como, proporcionou uma redução dos tamanhos dos microporos e um aumento da área superficial. Os espectros de infravermelho apresentaram bandas referentes aos grupos silanois.
\end{abstract}

Palavras-chave: Síntese, SBA-15, Etanol.

\section{INTRODUÇÃO}

Em 1998, um novo material foi sintetizado em condições fortemente ácidas $(\mathrm{pH}<2)$ utilizando copolímeros triblocos (Pluronic P123) como agentes direcionadores de estrutura. Esse material sintetizado tinha uma estrutura mesoporosa com um diâmetro de poros entre 46 e $300 \AA$, com alta área superficial, paredes com largas espessuras e com uma estabilidade térmica maior do que os materiais mesoporosos descobertos anteriormente como a MCM-41. A forma hexagonal bidimensional obtida desse material foi desenvolvido na Universidade de Santa Bárbara, nos Estados Unidos, e denominado SBA-15 (Santa Barbara Amorphous number 15). 
O pluronic P123 é formado por um bloco central de poli(óxido de propileno) - PPO e por blocos laterais de poli(óxido de etileno) - PEO, com formula molecular $\left(\mathrm{PEO}_{20} \mathrm{PPO}_{70} \mathrm{PEO}_{20}\right)(Z \mathrm{HAO}$ et al., 1998b). Direcionadores de estrutura como o P123 são moléculas anfifílicas, pois são constituídas de uma região hidrofóbica (PPO), não polar, e uma hidrofílica (PEO), polar. Moléculas anfifílicas se auto organizam em uma variedade de estruturas. A forma mais simples dessas estruturas é a micela. Micelas são agregados moleculares possuindo ambas as regiões estruturais, hidrofílica e hidrofóbica, que dinamicamente se associam espontaneamente em solução aquosa a partir de uma certa concentração micelar crítica (CMC), formando grandes agregados moleculares de dimensões coloidais. Esses agregados coloidais podem apresentar diferentes microestruturas: esférica, cilíndrica, planar, etc (OURIQUES, 2003; SOUSA, 2009). O etanol é um solvente polar prótico de forma molecular $\mathrm{C}_{2} \mathrm{H}_{5} \mathrm{OH}$, totalmente miscível em água em todas as proporções e solúvel na maior parte dos solventes orgânicos. É sem dúvida, o representante economicamente mais importante dos alcoóis alifáticos e como solvente perde apenas para a água, sendo empregado em quase todas as indústrias(SILVA e CAMPOS, 2013).

Segundo JOHANSSON, (2010), na síntese da SBA-15 num dado intervalo de temperatura o PPO é solúvel em etanol. Quando o co-solvente entra no núcleo hidrofóbico das micelas, o volume do núcleo se expande. O presente trabalho tem como objetivo avaliar a influência do etanol como co-solvente na formação da estrutura mesoporosa da peneira molecular SBA-15 em diferentes proporções.

\section{MÉTODOS}

A peneira molecular SBA-15 foi sintetizada baseada na metodologia descrita por ZHAO et al. (1998b), utilizando o etanol como co-solvente em diferentes proporções molares: 1TEOS: 0,017 P123:5,7 $\mathrm{HCl}$ : $173 \mathrm{H}_{2} \mathrm{O}: \mathrm{X}$ ETOH, em que $\mathrm{X}$ assumiu os seguintes valores 10, 20, 30 e 40. As amostras foram nomeadas segundo a Tabela 1 .

Tabela 1: nomenclatura utilizada para as amostras da peneira molecular SBA-15 em diferentes proporções de co-solvente etanol.

\begin{tabular}{cc}
\hline Nomenclatura & Proporção \\
\hline SBA-15_ET_10 & 10 \\
\hline SBA-15_ET_20 & 20 \\
\hline SBA-15_ET_30 & 30 \\
\hline SAB-15_Et_40 & 40 \\
\hline
\end{tabular}

Inicialmente, o Pluronic ${ }^{\circledR}$ P-123 foi dissolvido em uma solução contendo água, etanol e ácido clorídrico com concentração $2 \mathrm{M}$, sob agitação contínua e temperatura de 
$35^{\circ} \mathrm{C}$. Após a homogeneização do material, gotejou-se o TEOS na solução, e mantevese sob agitação a $35^{\circ} \mathrm{C}$ durante $20 \mathrm{~h}$. Em seguida, a amostra foi transferida para autoclaves que permaneceram na estufa durante $24 \mathrm{~h}$ a uma temperatura de $80{ }^{\circ} \mathrm{C}$. Posteriormente, foram realizadas lavagens com água deionizada para remoção do excesso do surfactante e logo após foi feita a secagem em estufa a $60^{\circ} \mathrm{C}$ durante $24 \mathrm{~h}$.

\subsection{Caracterizações}

Difração de raios-X (DRX): Para a determinação das fases cristalinas e dos parâmetros de arranjo hexagonal dos materiais sintetizados foi utilizada a difração de raios-X. Os valores dos espaçamentos interplanares $\left(\mathrm{d}_{(\mathrm{hkl})}\right)$ foram determinados a partir da lei de Bragg descrita a seguir:

$$
n \lambda=2 d_{(h k l) .} \operatorname{sen} \theta
$$

em que:

Comprimento de onda $(\lambda)=1,5418 \AA$;

$\mathrm{d}\left({ }_{\mathrm{hkl}}\right)$ : distância entre o índice de planos (hkl);

hkl: índice de Miller;

O parâmetro de rede, $\mathrm{a}_{0}$, foi calculado usando a equação 2:

$$
a_{0}=\frac{2 d_{100}}{\sqrt{3}}
$$

Microscopia eletrônica de Varredura (MEV): As analises foram realizadas no aparelho da Shimadzu SSX-55. Nesta técnica faz-se passar um feixe de elétrons através das lentes condensadoras e objetivas, executando uma varredura ao longo da amostra, enquanto um detector conta o número de elétrons secundários de baixa energia emitidos por cada ponto da superfície (NEWALKAR e KOMARMENI, 2002).

Infravermelho (FT-IR): A análise por espectroscopia de absorção na região do infravermelho foi utilizada com o objetivo de identificar qualitativamente as frequências vibracionais e suas respectivas atribuições referentes aos grupos funcionais presentes no material mesoporoso do tipo SBA-15, os espectros foram obtidos no infravermelho médio na faixa de $4000-600 \mathrm{~cm}^{-1}$.

\section{RESULTADOS E DISCUSSÕES}

Nos difratogramas das peneiras moleculares SBA-15 para diferentes proporções de co-solvente (etanol), apresentado na Figura 1, observa-se a obtenção dos três picos principais de difração, referentes aos planos cristalinos, cujos índices de Miller são (1 0 
0), ( $\left.\begin{array}{lll}1 & 1 & 0\end{array}\right)$ e $\left(\begin{array}{lll}2 & 0 & 0\end{array}\right)$. Esses sinais são característicos da rede hexagonal bem ordenada, conforme relatado por (Zhao et al., 1998 a e b).
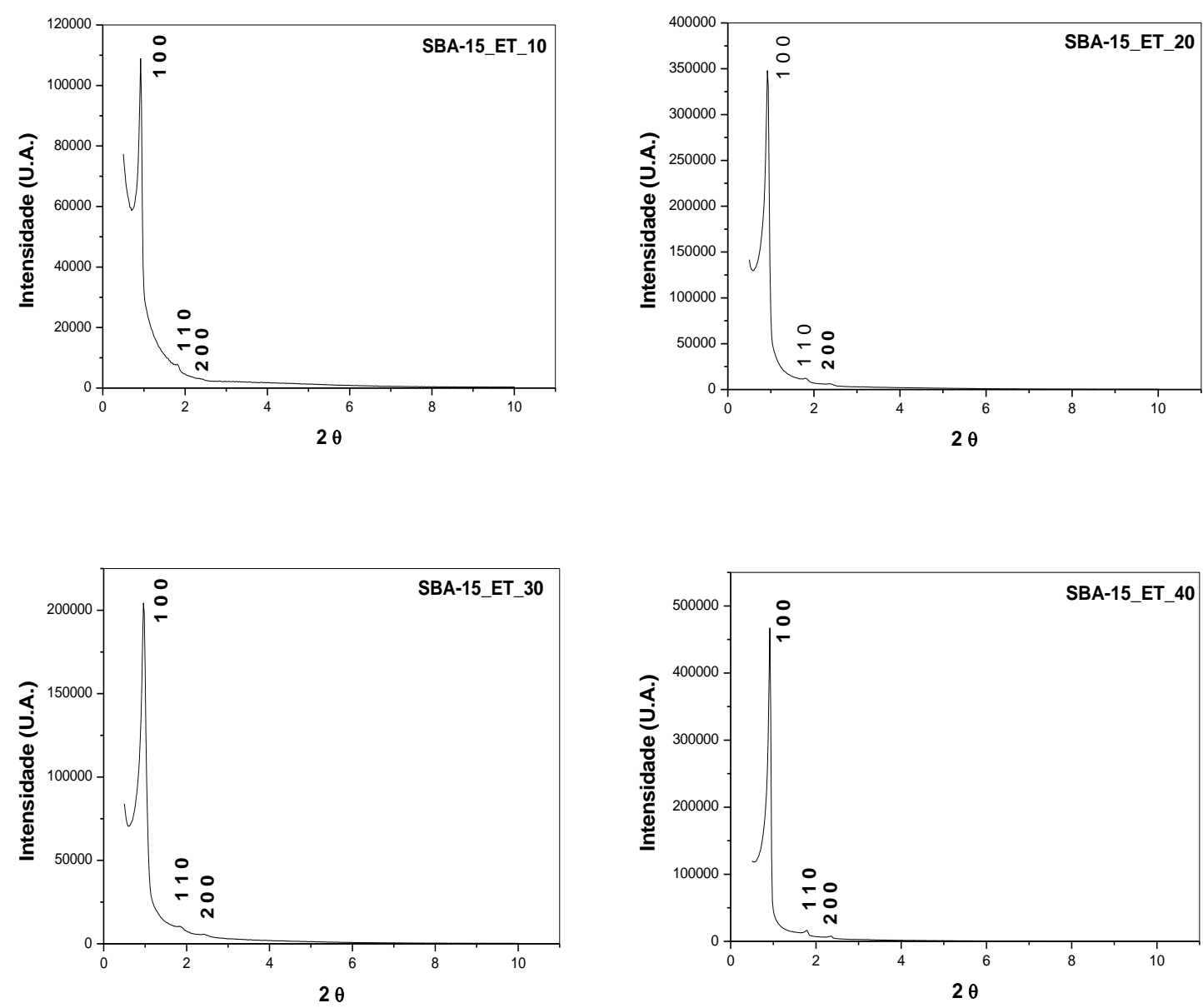

Figura 1: Difratometria de raios- $X$ das peneiras moleculares SBA-15 em todas as proporções molares de co-solvente etanol.

A medida que a proporção do co-solvente aumenta os picos correspondentes aos índices de 100 e 2000 tornam-se mais intensos e bem definidos, indicando um aumento no ordenamento estrutural da amostra. Os parâmetros cristalográficos das amostras estão apresentados na Tabela 1.

Tabela 1: Parâmetros cristalográficos das peneiras moleculares SBA-15 obtidas com diferentes proporções molares de etanol.

\begin{tabular}{|c|c|c|c|c|}
\hline Amostras & $2 \theta$ & h k l & $\mathbf{d}_{(\mathbf{1 0 0 )}}(\AA)$ & $\mathbf{a}_{0}(\AA)$ \\
\hline \multirow{3}{*}{ SBA-15_ET_10 } & 0,93 & 100 & 94,99 & 109,68 \\
\hline & - & 110 & & \\
\hline & 2,31 & 200 & & \\
\hline \multirow{2}{*}{ SBA-15_ET_20 } & 0,93 & 100 & 94,99 & 109,68 \\
\hline & 1,80 & 110 & & \\
\hline
\end{tabular}




\begin{tabular}{|c|c|c|c|c|}
\hline & 2,36 & 200 & & \\
\hline \multirow{3}{*}{ SBA-15_ET_30 } & 0,96 & 100 & 92,02 & 106,26 \\
\hline & 1,86 & 110 & & \\
\hline & 2,51 & 200 & & \\
\hline \multirow{3}{*}{ SBA-15_ET_40 } & 0,92 & 100 & 96,02 & 110,88 \\
\hline & 1,76 & 110 & & \\
\hline & 2,34 & 200 & & \\
\hline
\end{tabular}

De acordo com a Tabela 1 verificou-se para todas as amostras o pico 100 refletiu

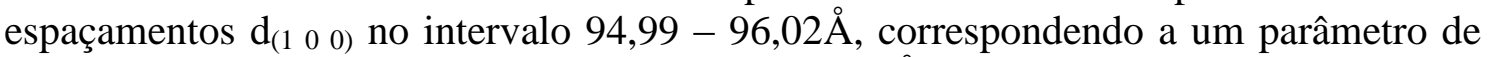
unidade de célula $\mathrm{a}_{0}$ no intervalo $109,68-110,88 \AA$, estes valores encontram-se em conformidade com a literatura descrita por (ZHAO,1998b).

Analisando as micrografias apresentadas na Figura 2 é possível identificarmos aglomerados de partículas esféricas com diâmetro de aproximadamente 1,92; 1,96 , 3,0 e $3,18 \mu \mathrm{m}$, separadas por espações vazios.

Com o aumento da proporção do co-solvente etanol os aglomerados de esferas tornam-se mais definidos e apresentam diâmetros maiores.

Pode-se observar que a adição do co-solvente etanol favorece a formação de partículas esféricas, pois o etanol diminui a taxa de hidrólise de TEOS e reduz a polaridade da solução aquosa, fazendo com que seja possível sintetizar esta morfologia (KOSUGE e SINGH, 2001). Esta morfologia é de interesse para aplicações como sistemas de entrega de medicamentos ou armazenamento de gás (JOHANSSON, 2010).

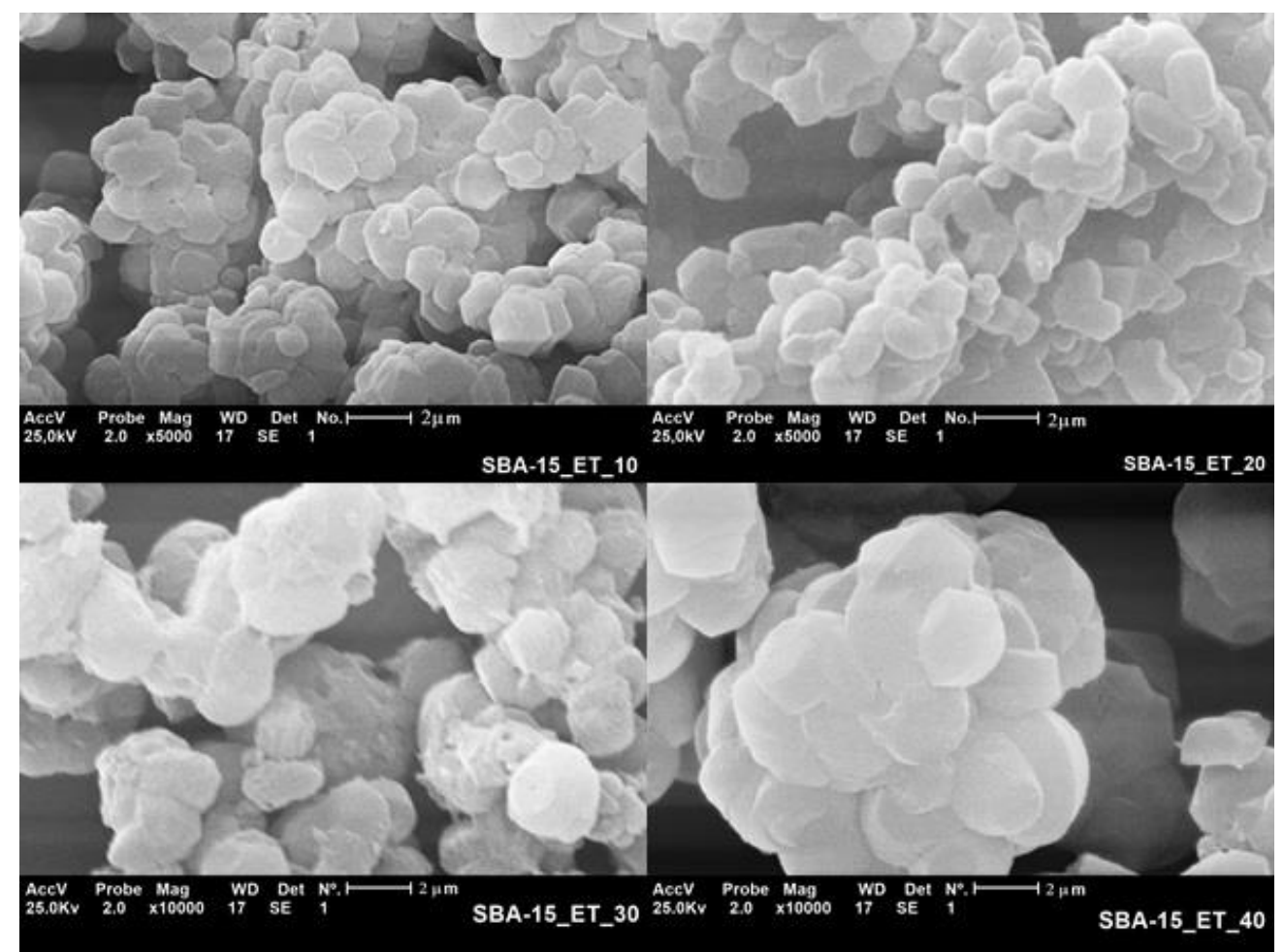

Figura 2: Micrografias das peneiras moleculares SBA-15 obtidas em diferentes proporções molares do co-solvente etanol. 
Os espectros de absorção na região do infravermelho na faixa de 600 a $4000 \mathrm{~cm}^{-1}$, da peneira molecular SBA-15 para diferentes proporções molares de co-solvente etanol encontra-se na Figura 3. Os espectros de infravermelho para todas as proporções de cosolvente apresentam as mesmas bandas com variações de absorbância mínimas. Observa-se bandas típicas referentes a estiramentos assimétricos da ligação $\mathrm{Si}-\mathrm{O}-\mathrm{Si}$ em torno de 1051,64 $\mathrm{cm}^{-1}$, e em 796,99 $\mathrm{cm}^{-1}$ devido a estiramentos simétricos das ligações $\mathrm{Si}-\mathrm{O}$, fato bastante comum em silicatos e aluminosilicatos (WANG et al., 2005). O pico em torno de $940 \mathrm{~cm}^{-1}$ pode ser atribuído a vibrações $\mathrm{Si}-\mathrm{OH}$ geradas pela presença de sítios defeituosos na estrutura.
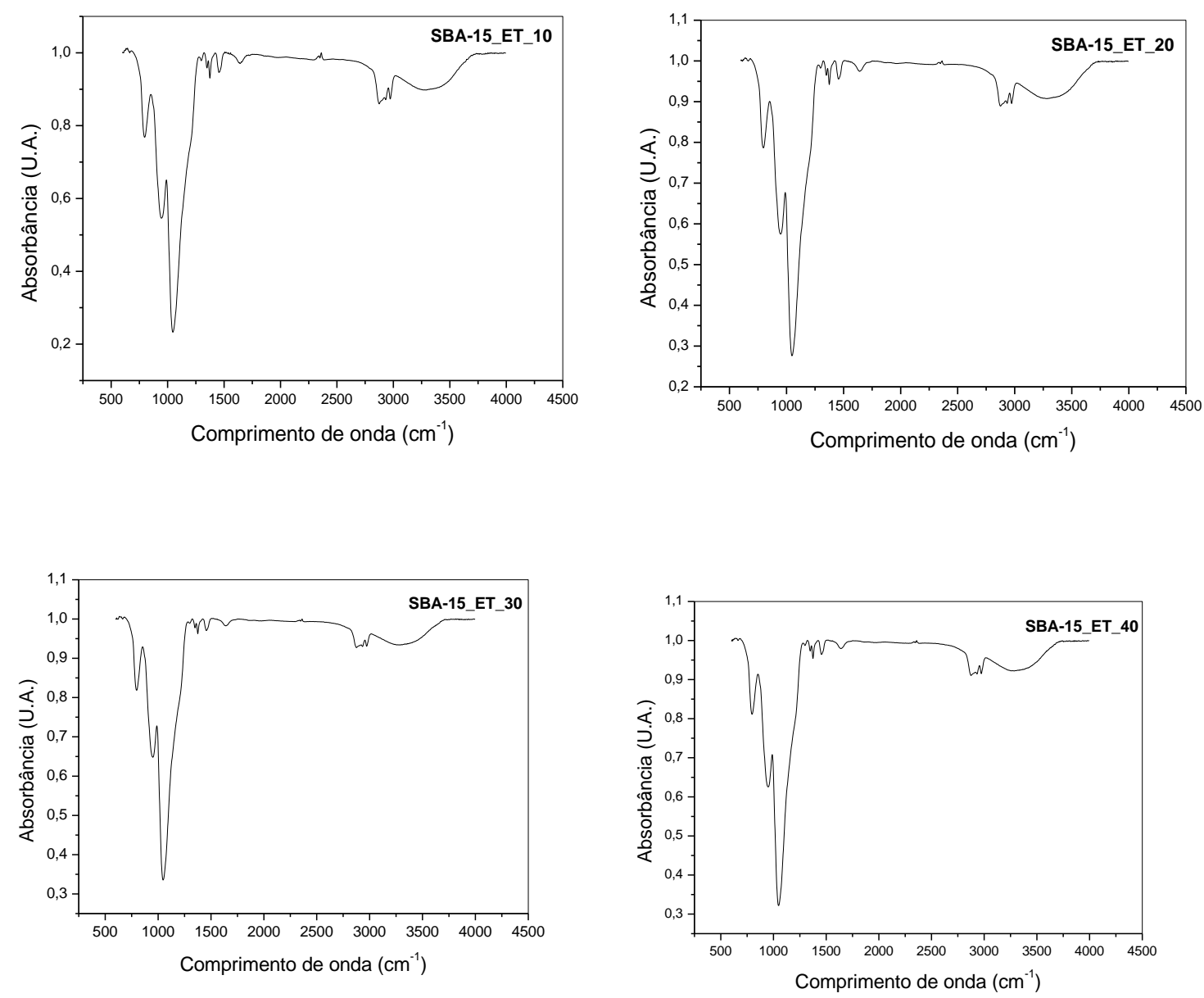

Figura 3: Espectros de infravermelho das amostras da peneira molecular SBA-15 para todas proporções molares do co-solvente etanol.

A Tabela 2 mostra um resumo dos dados relativos as frequências vibracionais observadas e suas respectivas atribuições para o espectro de infravermelho da peneira molecular SBA-15 para todas as proporções de co-solvente etanol. 
Tabela 2: Resumo das atribuições para os espectros na região do infravermelho para a peneira molecular SBA-15 em todas as proporções de co-solvente etanol.

\begin{tabular}{|c|c|}
\hline Número de onda $\left(\mathrm{cm}^{-1}\right)$ & Atribuição \\
\hline $3013,46-3314,90$ & $\begin{array}{c}\text { Estiramento dos grupos hidroxilas } \\
\text { internos e externos na estrutura } \\
\text { mesoporosa. }\end{array}$ \\
\hline 1640,00 & Água adsorvida na superfície do material \\
\hline 1458,90 & $\begin{array}{c}\text { Deformação da ligação }-\left(\mathrm{CH}_{2}\right)_{\mathrm{n}} \text { da cadeia } \\
\text { do direcionador } \mathrm{P} 123\end{array}$ \\
\hline 1374,32 & $\begin{array}{c}\text { Deformação da ligação } \mathrm{C}-\mathrm{O}-\mathrm{C} \text { do } \\
\text { direcionador } \mathrm{P} 123\end{array}$ \\
\hline 1051,64 & $\begin{array}{l}\text { Estiramentos assimétricos das ligações } \\
\qquad \mathrm{Si}-\mathrm{O}-\mathrm{Si}\end{array}$ \\
\hline 949,60 & $\begin{array}{c}\text { Vibrações } \mathrm{Si}-\mathrm{OH} \text { de sítios defeituosos na } \\
\text { estrutura }\end{array}$ \\
\hline 796,99 & $\begin{array}{l}\text { Estiramentos simétricos das ligações } \mathrm{Si}- \\
\mathrm{O}\end{array}$ \\
\hline
\end{tabular}

\section{CONCLUSÃO}

Diante dos difratogramas obtidos verificou-se que na síntese da SBA-15 a medida que a proporção de co-solvente etanol aumentou ocorreu a formação de um material mais organizado estruturalmente devido a um aumento na intensidade dos picos característicos dos planos $\left(\begin{array}{lll}1 & 1 & 0\end{array}\right)$ e $\left(\begin{array}{lll}2 & 0 & 0\end{array}\right)$. As micrografias mostraram que a medida que a proporção do co-solvente etanol aumentou os aglomerados de esferas tornaram-se mais definidos e apresentaram diâmetro de partículas esféricas aglomeradas maiores. Os espectros de infravermelho para todas as proporções de co-solvente detectaram bandas características de material mesoporoso do tipo SBA-15.

\section{REFERÊNCIAS}

JOHANSSON, E. M. Controlling the Pore Size and Morphology of Mesoporous Silica. Nanostructured Materials Division - Department of Physics, Chemistry and Biology (IFM). Linköping University,2010. Tese, 97p.

KOSUGE, K.; SINGH, P.S. Mesoporous Silica Spheres Via L-Alkylamine Templating Route. Chem. of M.. 13 (2001) 2476-2482.

NEWALKAR, B. L.; KOMARNENI, S., Simplified synthesis of micropore-free mesoporous silica, SBA-15, under microwave-hydrothermal conditions. Chem. Com. (Cambridge, United Kingdom) 2002, (16), 1774-1775. 
OURIQUES, G. R. Estudo da Interação entre Moléculas Anfifílicas e Água no Sistema Binário APFO/H2O por Métodos Computacionais. In: $3^{\text {a }}$ Semana de Ensino, Pesquisa e Extensão - UFSC, 2003.

SOUSA, A. Híbridos de Gel Polimérico em Sílica Mesoporosa Estruturalmente Ordenada para Liberação Controlada de Fármacos. Belo Horizonte-MG. Programa de Pós-Graduação em Engenharia Química. UFMG, 2009. Tese de Doutorado, 159 p.

SILVA, W. A. da e CAMPOS, V. R. Métodos de Preparação Industrial de Solventes e Reagentes Químicos: Etanol. Revista Virtual de Química. v.5, n. 5, 2013. Disponível em: < http://www.uff.br/RVQ/index.php/rvq/article/viewFile/457/376 >. Acesso em: 20 de abril de 2014.

WANG, X. et al. Characterization and catalytic properties of tin containing mesoporous silicas prepared by different methods. Jour. of Mol. Cat. A: Chemical, 238, $185-191,2005$.

ZHAO, D.; HUO, Q.; FENG, J.; CHMELKA, B. F.; STUCKY, G. D. Nonionic triblock and star diblock copolymer and oligomeric surfactant syntheses of highly ordered, hydrothermally stable, mesoporous sílica structures. In: Jour. of the American Chem. Soc., v. 120, p. 6024-6036, 1998a.

ZHAO, D.; FENG, J.; HOU, Q.; MELOSH, N.; FREDRICKSON, G. H.; CHMELKA, B. F.; STUCKY, G. D. Triblock copolymer syntheses of mesoporous silica with periodic 50 to 300 angstrom pores. Science, v.279. n.5350, p.548 - 552, 1998 b. 